\title{
Electronic states on a fractal: Exact Green's-function renormalization approach
}

\author{
R. F. S. Andrade \\ Instituto de Fisica, Universidade Federal da Bahia, 40210 Salvador, Brazil \\ H. J. Schellnhuber \\ Fachbereich Physik and Institut für Chemie und Biologie des Meeres, Universität Oldenburg, D-2900 Oldenburg, \\ Federal Republic of Germany \\ (Received 5 March 1991)
}

\begin{abstract}
A nontrivial tight-binding model for electron dynamics on the fractal Koch curve is investigated within the framework of the Green's-function formalism. The key result is the construction of a multiple exact renormalization group that allows one to derive all the rather unusual properties of the model. This group is generated by four nonequivalent decimation operations, which define distinct transformation rules for the 48 relevant parameters to be renormalized. The calculation of the density of states confirms the crucial results that were obtained recently using transfer-matrix methods: local self-affinity, dense gap structure, and singular electronic levels with infinite degeneracy. This demonstrates that the Green's-function approach is not inferior to other techniques even in topologically one-dimensional situations.
\end{abstract}

\section{INTRODUCTION}

Green's-function methods have widespread applications in physics. In particular, they represent powerful tools for the evaluation of various properties of large quantum systems. The formalism is seldom capable of deriving exact results, but it generally offers a systematic way to obtain reasonable-or even excellentapproximations on the basis of perturbative series.

A very important system, which has been investigated extensively by this approach, is the problem of one particle in a nonperiodic potential that is bounded everywhere in space. Such a system is expected to mimic the electron dynamics in solids lacking perfect crystalline order. For many years the "hot or dirty crystal" picture has dominated the field: Aperiodicity was achieved by adding random functions to a periodic effective potential. ${ }^{1-7}$ Ingenious Green's-function techniques have been devised to solve the resulting problems, but the analytical and numerical difficulties that must be surmounted to obtain conclusive results are formidable. ${ }^{8}$

In the past decade, however, a new philosophy has emerged: The aperiodic solid is modeled as a deterministically disordered substrate for quasiparticle motion. This means that hierarchical ${ }^{9}$ or quasiperiodic $^{10-14}$ potentials are studied, which are supposed to interpolate between simple crystalline order and complete stochasticity. Such models have the virtue of being exactly or approximately invariant with respect to the operations of some renormalization group (RG).

By exploring this property it has been demonstrated that deterministically disordered systems do not only simulate important familiar aspects of randomly disordered ones: they also possess a number of very intricate and novel features (critical states, singular-continuous spectral components, etc.) that may or may not be present in the latter type of models. These features were mainly detected by transfer-matrix methods (TMM), which are well-suited for the implementation of the RG.

At first glance, Green's-function techniques do not appear to be good candidates for the same job, although they are more easily extended to higher dimensions than the TMM. The reason is that any perturbative treatment, like the Green's-function decimation scheme introduced by Gonçalves da Silva and Koiller, ${ }^{7}$ must fail to distinguish between, say, absolutely continuous and singularcontinuous spectral measures. The Green's-function formalism becomes, however, a competitive (and in some respects even superior) approach to deterministically disordered systems, if it can be made exact whenever other methods do achieve this.

The intention of our paper is to support this conjecture by showing how a limit-periodic model with rather spectacular properties can be solved without approximations by appropriate Green's-function methods. This model describes electron hopping on the Koch fractal and was recently analyzed by the authors by means of TMM. ${ }^{15}$ Very few examples of exact Green's-function renormalization schemes for tight-binding Hamiltonians can be found in the literature. Interesting cases have been studied by Langlois, Trembley, and Southern ${ }^{9}$ and by Ashraff and Stinchcombe ${ }^{16}$ (see also Ref. 14). Our work differs from theirs in two main respects.

(1) Electron dynamics on the fractal substrate considered here is much richer than those encountered in hierarchical or quasiperiodic structures like the Fibonacci chain: ${ }^{12,14}$ It was demonstrated in Ref. 15 that all possible types of spectral components and localization behavior are present, that a host of mobility edges exist, and that an infinity of quantum levels condense for certain choices of the system parameters to form a superlocalized phase. The correct Green's-function approach 
has to account for all these phenomena.

(2) The exact Green's-function renormalization schemes of Refs. 9 and 16 are, like ours, devised in the spirit of the Gonçalves da Silva-Koiller decimation approach. But they either restrict themselves to pivotal sites to obtain information on the density of states and other quantities of interest, or they make use of compositional averages. Our approach takes explicitly into account all sites on the fractal, which actually contribute differently to the electronic properties of the model. This can only be achieved by extending the standard renormalization machinery: Our system turns out to be exactly invariant with respect to a family of nonequivalent dilation-symmetry operations, which define separate 48parameter renormalization subgroups for the Green's coefficients.

The material in our paper is organized in the following way: In Sec. II we present the model, briefly review its pertinent properties as demonstrated in Ref. 15, and introduce the formalism. In Sec. III the explicit Green'sfunction RG for the system is constructed taking great pains with the treatment of all nonequivalent classes of sites. Section IV summarizes crucial numerical results produced by the exact renormalization scheme, and Sec. $\mathrm{V}$ concludes the paper with a short discussion.

\section{MODEL AND GREEN'S-FUNCTION FORMALISM}

We consider the tight-binding Hamiltonian describing nearest-neighbor hopping on the fractal Koch curve, which has been introduced in Ref. 15. Probably the best way to evaluate the physical properties of this system is to use periodic approximations of order $N$ for the "terragon," as illustrated in Fig. 1.

The crucial feature of our model, which gives rise to quite unusual properties, is the possibility of electron hopping across the folded chain to nearest neighbors in the Euclidean sense. The strength of this interaction is measured by the parameter $\lambda$, while nearest-neighbor hopping along the chain is fixed to unit rate.

The $N$ th periodic approximation is governed by the Hamiltonian $H_{N}$. For $N \geq 1$ it is given by

$$
\begin{array}{r}
H_{N}=\sum_{n \in \mathbb{Z}}[|n\rangle\langle n+1|+| n\rangle\langle n-1| \\
+\lambda t_{N}(n)(|n-1\rangle\langle n+1| \\
+|n+1\rangle\langle n-1|)],
\end{array}
$$

where the effective next-nearest-neighbor interaction is specified as follows:

$$
\begin{aligned}
& t_{N}(n)=\delta(0, n)+\sum_{s=1}^{N-1} \delta\left(\frac{1}{2} 4^{s}, n\left(\bmod 4^{s}\right)\right) \\
& \qquad-\frac{1}{2} 4^{N}<n \leq \frac{1}{2} 4^{N} ; \\
& t_{N}\left(n+4^{N}\right)=t_{N}(n) \text { for all } n \in \mathbb{Z} .
\end{aligned}
$$

Note that the fractal structure of our model enforces an exponentially growing sequence of approximation periods, namely $\left\{4^{N} \mid N \in \mathbb{N}\right\}$.

The $N$ th-order Green's-function $G_{N}$ associated with $H_{N}$ is formally defined by $\left(E-H_{N}\right) G_{N}(E)=\mathbb{1}$. It is explicitly determined by the following set of equations:

$$
\begin{aligned}
\left\langle n\left|\left(E-H_{N}\right) G_{N}\right| m\right\rangle & =\sum_{k \in \mathbb{Z}}\left\langle n\left|\left(E-H_{N}\right)\right| k\right\rangle\left\langle k\left|G_{N}\right| m\right\rangle \equiv \sum_{k \in \mathbb{Z}}\left[E \delta(n, k)-H_{n k}^{(N)}\right] G_{k m}^{(N)} \\
& =-\lambda t_{N}(n+1) G_{n+2, m}^{(N)}-G_{n+1, m}^{(N)}+E G_{n m}^{(N)}-G_{n-1, m}^{(N)}-\lambda t_{N}(n-1) G_{n-2, m}^{(N)} \\
& =\delta(n, m), \quad n, m \in \mathbb{Z} .
\end{aligned}
$$

$N=0$ :
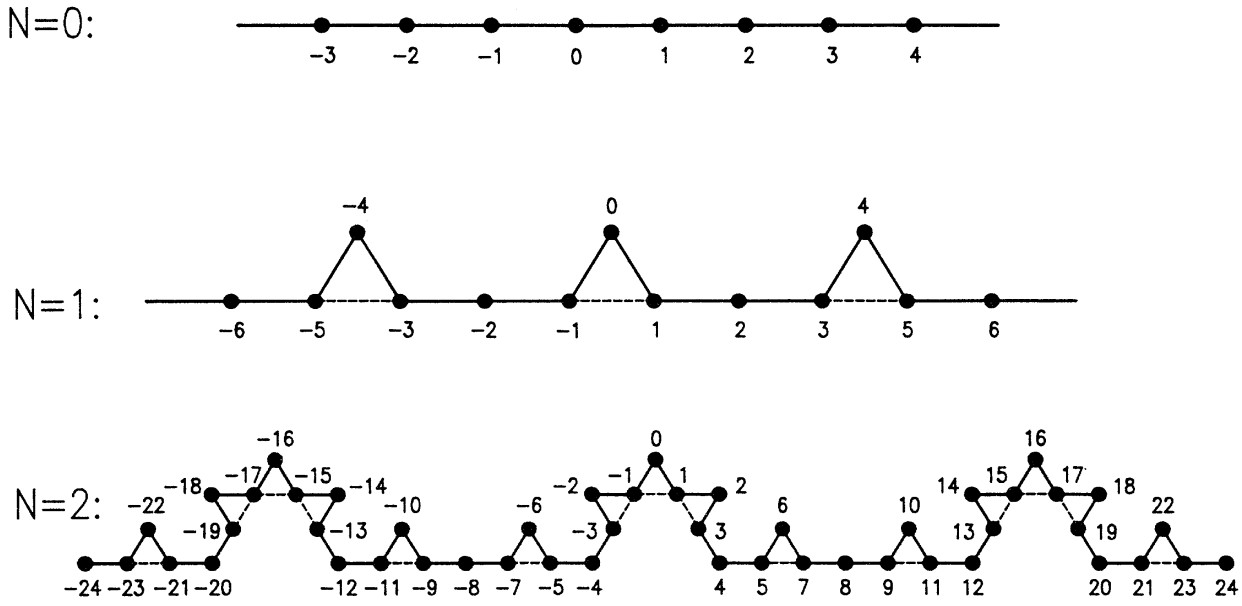

FIG. 1. The first three stages in the construction of the Koch tight-binding Hamiltonian by periodic approximation. 
$G_{N}$ contains all information about the $N$ th approximation of the quantum fractal. In particular, the density of states (DS) $\rho_{N}(E)$ can be obtained as

$$
\rho_{N}(E)=-\frac{1}{\pi} \lim _{S \rightarrow \infty} \operatorname{Im}\left[\frac{1}{S} \sum_{s=1}^{S} G_{s s}^{(N)+}(E)\right],
$$

where

$$
G_{s s}^{(N)+}(E)=\lim _{\epsilon \rightarrow 0} G_{s s}^{(N)}(E+i \epsilon) .
$$

The corresponding integrated density of states (IDS) $\kappa_{N}(E)$ is defined by

$$
\kappa_{N}(E)=\int_{-\infty}^{E} \rho_{N}(\bar{E}) d \bar{E}
$$

The full sequence of Green's functions $\left\{G_{N}(E) \mid N \in \mathbb{N}\right\}$ will reveal all the particular spectral features which were discovered in Ref. 15. A typical property of our limitperiodic model system ${ }^{17}$ is the local self-affinity ${ }^{18}$ of the DS and the IDS, which is directly linked to the presence of "exotic states"12,13 displaying dilational symmetry and power-law (de)localization. Another generic aspect is the fact that the spectrum is nowhere dense due to the perpetual subband splitting that takes place in the sequence of approximations $\left\{\rho_{N}(E) \mid N \in \mathbb{N}\right\}$ to the limit DS.

A most striking and unusual feature of our fractalhopping dynamics, however, is the appearance of levels possessing infinite degeneracy and finite spectral weights when the cross-hopping rate $\lambda$ is chosen as $3^{-1 / 2}, 2^{-1 / 2}$, or 1. These levels are populated by superlocalized or "molecular" states. ${ }^{19}$

In Sec. IV we will show in an exemplary way how the Green's-function approach reproduces such subtle effects obtained by TMM. To that end the full scheme has to be employed: The familiar restriction to the evaluation of just one (conveniently chosen) diagonal coefficient $G_{s s}^{(N)+}(E)$ in Eq. (4) is not correct here, because different sites on the fractal will contribute differently to the DS. In view of the lack of any type of translational symmetry in the system, the Green's function cannot be averaged.

Let us get a feeling for the consequences of this complication. We first consider the odd-numbered sites on the Koch curve, which cannot be bypassed via direct interaction between their left and right neighbors on the fractal (see Fig. 1). These sites therefore represent nodes and can be used to replace the system by an effective onedimensional model with nearest-neighbor interactions of two different types. This is achieved by a decimation scheme that retains only one out of four sites and introduces new "lumped" interactions according to the following rule:

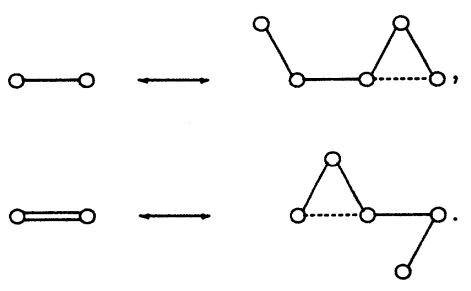

This recipe preserves just one-half of the odd sites, namely those with labels $4 t-1, t \in \mathbb{Z}$. Another possible decimation procedure would eliminate all sites except those with labels $4 t+1, t \in \mathbb{Z}$. As the structure is invariant with respect to the index reflection $n \rightarrow-n$, we can restrict ourselves to one particular choice.

Our first decimation step is equivalent to the description of the system in terms of the matrix products $H G$ and $F F$ introduced in Ref. 15 . Note that the $N$ th approximation to the fractal limit structure is converted in this step into a $4^{N-1}$-periodic arrangement of single and double interactions.

Considering now the even-numbered sites, we observe that a subset of them appears in a local topology, which allows the right and left (odd-numbered) neighbors on the curve to interact directly with hopping rate $\lambda$. This fact prevents us from performing the same decimation scheme that has been employed to the odd sites. If we tried to carry out this procedure by keeping one-half of the even sites, then next-nearest-neighbor interactions would emerge and destroy any hope of coming up with a compact RG. The difficulty can be circumvented, however, by referring the contributions of the even sites to the DS to their odd neighbors, which can be treated in the way described above. All this will be discussed in the following section.

\section{EXACT RENORMALIZATION GROUP}

The most powerful RG techniques for the evaluation of the Green's functions of tight-binding models are based on the idea of repeatedly eliminating the sites neighboring a pivotal site $\bar{n}$ and summing up their contributions in renormalized interaction coefficients for the remaining sites. ${ }^{6,7,9,11}$ Any such decimation procedure is conceptually straightforward for each element $G_{\bar{n} m}$. It is particularly easy to perform in the diagonal case, i.e., $m=\bar{n}$, in which we are mainly interested.

Note, however, that a RG scheme is only useful when the decimated system has the same structure as the original one; then the procedure can be repeated over and over again until the site $\bar{n}$ becomes virtually decoupled from its neighbors. For systems with random disorder, this condition is certainly not fulfilled. The strategy was, nevertheless, successfully applied to such models by resorting to configurational averages (see, e.g., Ref. 7); then $G_{00}$ is used as a representative for all $G_{\bar{n} \bar{n}}$. The price to be paid is reduced accuracy and the risk of missing subtle but characteristic features like critical wave functions.

By way of contrast, an exact RG scheme can be devised for our fractal tight-binding system. As already mentioned above, there is also a price to be paid for this rigor: As fractals do not possess translational symmetry (be it exact or statistical), all $G_{\bar{n} \bar{n}}$ may contribute differently to the DS. One immediately realizes that the decimation procedure with pivotal site $\bar{n}$ defines an individual sequence of renormalization operations which are not necessarily identical to the sequences associated with other sites. In our case reflection symmetry only guaran- 
tees that $-\bar{n}$ generates the same scheme as $\bar{n}$. We have to distinguish, in particular, odd and even sites.

\section{A. Odd sites}

In the preceding section we indicated diagrammatically how our fractal system can be reduced to a linear one with two kinds of next-neighbor interactions. This scheme, as defined by rule (7), results in the decimation of all even sites and half of the odd sites. It is of practical use only if we are able to derive explicit relations between the new single and double interactions and the model parameters. We observe, however, that the expressions for these interactions are not unique.

Indeed, let us consider the complex energy $z=E+i \epsilon$ and define alternative coefficients of the system by

$$
x=\frac{1}{z}, \quad x_{l}=\lambda x, \quad g=\frac{1}{z}
$$

With these definitions Eq. (3) becomes

$$
\begin{aligned}
G_{n m}^{(N)}= & g \delta(n, m)+x_{l} t_{N}(n+1) G_{n+2, m}^{(N)}+x G_{n+1, m}^{(N)} \\
& +x G_{n-1, m}^{(N)}+x_{l} t_{N}(n-1) G_{n-2, m}^{(N)} .
\end{aligned}
$$

We can now eliminate all sites except those labeled as $4 t-1, t \in \mathbb{Z}$, from this set of equations and determine the new interaction coefficients connecting the remaining sites. It turns out, in particular, that the value for single-bond interactions between a pivotal site $4 \bar{t}-1$ and its left neighbor $4 \bar{t}-5$ depends on the type of bonding to the right neighbor $4 \bar{t}+3$.

Therefore, we must refine our definitions of interactions and our site classification by considering contextsensitive expressions, which take into account at least the left and right links of a given site. A first natural identification leads to the following site classification:

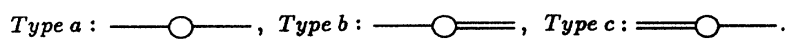

Yet this identification does the job only for the first decimation step. In the following stages of the RG scheme the classification (10) leads to contradictions: We may end up with two different renormalized expressions for the same entity. This would spoil our objective of obtaining for the decimated system the same structure as for the original one.

There exists, however, a sufficiently rich classification scheme for the site types, which also takes into account the bonds of the left and right next neighbors of the site in question. This scheme defines six classes according to the following rule:

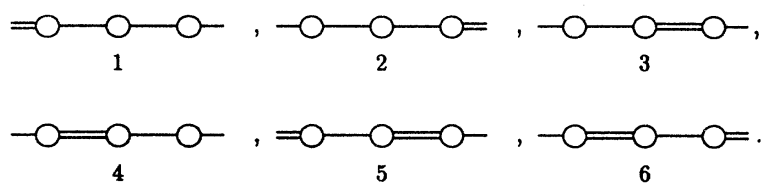

For $i \in\{1,2,3,4,5,6\}$ let us denote by $d_{i}$ and $e_{i}$ the interaction coefficients of a given site with its left and right neighbor, respectively. The renormalized value of the inhomogeneity $g$ also turns out to depend explicitly on the type of the site considered; therefore, it will be called $g_{i}$. Some straightforward algebra yields the expressions for these new entities in terms of the old parameters $x, x_{l}$, and $g$ as a function of site type. The derivation is sketched in Appendix A, while the results are summarized in Table I.

This first, rather laborious, decimation step prepares the ground for a straightforward and unique renormalization scheme embracing all subsequent steps. When deriving the pertinent renormalization rules we have to be aware of three crucial points.

(i) In spite of the necessity of introducing site-type dependent bonds, the pictorial decimation scheme, operating with single and double bonds only, is very convenient in the further process. Thus we will continue to identify single and double bonds and will work out how new bonds are related to old ones.

(ii) Depending on the position of the pivotal site in the system, one has to choose between four different consistent ways of defining new single and double bonds by pictorial decimation. They are described by the following majority rules:

TABLE I. Interaction coefficients $e_{i}, d_{i}$, and $g_{i}$ after the first decimation as a function of sites types $i=1, \ldots, 6$. The quantities $D_{1}$ and $D_{2}$ are defined as $D_{1}=1-4 x^{2}+2 x^{4}-x_{l}\left(x_{l}+2 x^{2}\right)$, $D_{2}=1-4 x^{2}+2 x^{4}-2 x_{l}\left(x_{l}+2 x^{2}\right)$.

\begin{tabular}{cccc}
\hline \hline & & Coefficients & \\
Site type & $d_{i}$ & $e_{i}$ & $g_{i}$ \\
\hline 1 & $x^{2}\left(x^{2}+x_{l}\right) / D_{1}$ & $x^{2}\left(x^{2}+x_{l}\right) / D_{1}$ & $g\left(1-2 x^{2}\right) / D_{1}$ \\
2 & $x^{2}\left(x^{2}+x_{l}\right) / D_{1}$ & $x^{2}\left(x^{2}+x_{l}\right) / D_{1}$ & $g\left(1-2 x^{2}\right) / D_{1}$ \\
3 & $x^{2}\left(x^{2}+x_{l}\right) / D_{2}$ & $\left(x^{2}+x_{l}\right)^{2} / D_{2}$ & $g\left(1-2 x^{2}\right) / D_{2}$ \\
4 & $\left(x^{2}+x_{l}\right)^{2} / D_{1}$ & $x^{2}\left(x^{2}+x_{l}\right) / D_{1}$ & $g\left(1-2 x^{2}\right) / D_{1}$ \\
5 & $x^{2}\left(x^{2}+x_{l}\right) / D_{2}$ & $\left(x^{2}+x_{l}\right)^{2} / D_{2}$ & $g\left(1-2 x^{2}\right) / D_{2}$ \\
6 & $\left(x^{2}+x_{l}\right)^{2} / D_{1}$ & $x^{2}\left(x^{2}+x_{l}\right) / D_{1}$ & $g\left(1-2 x^{2}\right) / D_{1}$ \\
\hline \hline
\end{tabular}




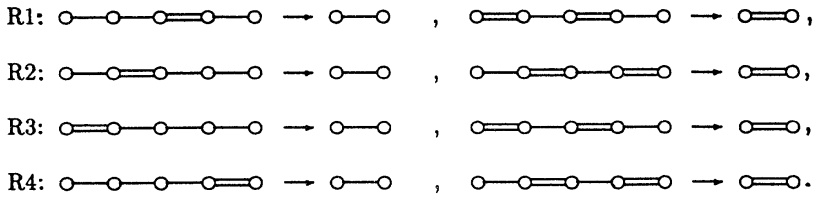

(iii) The type of preserved site is, in general, changed during a decimation step. The new type of a given site is determined by the specification of the majority rule (12), and by the old type of the site itself and its four neighbors to the right and the left prior to decimation.

In summary, we find that we have to distinguish a total of 24 different situations, which are characterized by a particular string of nine successive old site type numbers and the new type of the central site as a function of the decimation rule employed. All the information needed for the RG is gathered in Table II. Its use is explained in Appendix B.

Table II has to be consulted over and over again in the course of repeated renormalization. In principle, the complete series of decimation rules and site types associated with a particular initial site of the model can only be constructed by tracking down the evolution of each single site on the curve. Fortunately the hierarchical structure of the problem makes it possible to derive certain general prescriptions from the exemplary inspection of the renormalization series generated by the specific sites (see also Sec. II C). The following construction rules can be established.

(a) If the system is a periodic approximation of order $N$, then we are left with a total of $4^{N-1}$ different sites after the first decimation step as described above. $\mathcal{N}(N)$ members of these surviving sites are of types $1,2,3$, and 4 , and $2 \overline{\mathcal{N}}(N)$ are in classes 5 and 6 , respectively. The numbers $\mathcal{N}(N)$ and $\overline{\mathcal{N}}(N)$ are given by

$$
\begin{aligned}
& \mathcal{N}(N)=\frac{1}{3}\left(2 \times 4^{N-2}+1\right), \\
& \overline{\mathcal{N}}(N)=\frac{1}{3}\left(4^{N-2}-1\right) .
\end{aligned}
$$

Formula (13) also accounts for the abundance of individual site types after all the following decimations, provided that the argument of $\mathcal{N}$ and $\overline{\mathcal{N}}$ is reduced by 1 in each renormalization step.

(b) The sites of types $1,2,3$, and 4 will be preserved exclusively by decimations of types $R 1, R 2, R 3$, and $R 4$, respectively. The sites of type 5 are preserved by either rule $R 1$ or $R 3$, while the sites of type 6 will be preserved by rule $R 2$ or $R 4$ (see Table II). Moreover, the same amount of sites of types 5 and 6 will be preserved by the two different decimation schemes. For certain purposes it is useful to refine the classification of the sites of types 5 and 6 as $5 . R 1$ or $5 . R 3$, and $6 . R 2$ or $6 . R 4$, respectively, to indicate clearly which type of decimation it will undergo.

(c) There is a universal law for the new type distribution of the surviving sites as a function of old site types. This law is illustrated in Table III.

The successive application of these rules is sufficient to generate all sequences starting from any periodic approximation $N$. The knowledge of these sequences and of Table II allows us to exactly renormalize the interaction coefficients for all $4^{N-1}$ sites.

The recursion relations for these coefficients and for the inhomogeneities can be derived in a perfectly general way by considering an arbitrary site $p$ and the associated local string of equations

$$
\begin{aligned}
& G_{p-3}=d_{i(p-3)} G_{p-4}+e_{i(p-3)} G_{p-2}, \\
& G_{p-2}=d_{i(p-2)} G_{p-3}+e_{i(p-2)} G_{p-1}, \\
& G_{p-1}=d_{i(p-1)} G_{p-2}+e_{i(p-1)} G_{p}, \\
& G_{p}=d_{i(p)} G_{p-1}+e_{i(p)} G_{p+1}+g_{i(p)}, \\
& G_{p+1}=d_{i(p+1)} G_{p}+e_{i(p+1)} G_{p+2}, \\
& G_{p+2}=d_{i(p+2)} G_{p+1}+e_{i(p+2)} G_{p+3}, \\
& G_{p+3}=d_{i(p+3)} G_{p+2}+e_{i(p+3)} G_{p+4} .
\end{aligned}
$$

For the sake of simplicity we have omitted in these equations both the approximation index $N$ and the second label $p$ of the Green's-function coefficients. Elimination of the sites $p-3, p-2, p-1, p+1, p+2$, and $p+3$ from (14) yields one single equation, which connects $G_{p}$ to $G_{p-4}$ and $G_{p+4}$ and defines the parameter renormalization group:

$$
\begin{array}{r}
d_{i^{\prime}(p)}^{\prime}=D^{-1} d_{i(p)} d_{i(p-1)} d_{i(p-2)} d_{i(p-3)}\left(1-d_{i(p+3)} e_{i(p+2)}\right. \\
\left.-d_{i(p+2)} e_{i(p+1)}\right), \\
e_{i^{\prime}(p)}^{\prime}=D^{-1} e_{i(p+3)} e_{i(p+2)} e_{i(p+1)} e_{i(p)}\left(1-d_{i(p-1)} e_{i(p-2)}\right. \\
\left.-d_{i(p-2)} e_{i(p-3)}\right),
\end{array}
$$$$
g_{i^{\prime}(p)}^{\prime}=D^{-1} g_{i(p)}\left(1-d_{i(p-1)} e_{i(p-2)}-d_{i(p-2)} e_{i(p-3)}\right)
$$$$
\times\left(1-d_{i(p+3)} e_{i(p+2)}-d_{i(p+2)} e_{i(p+1)}\right) .
$$

Here $D$ is given by

TABLE II. New site types as a function of the old types of the preserved site and its eight next neighbors (characterized by nine-digit strings), and of the bond-renormalization rule employed.

\begin{tabular}{ccccc}
\hline \hline & \multicolumn{4}{c}{ Decimation type } \\
New site type & 1 & 2 & 3 & 4 \\
\hline 1 & 123412341 & 654123412 & 541234123 & 412341234 \\
2 & 123412365 & 234123412 & 341234123 & 412341236 \\
3 & 123656541 & 234123656 & 341236565 & 412365654 \\
4 & 565412341 & 236565412 & 365654123 & 656541234 \\
5 & 123656541 & 654123656 & 541236565 & 412365654 \\
6 & 565412365 & 236565412 & 365654123 & 656541236 \\
\hline \hline
\end{tabular}


TABLE III. Type distribution of sites retained after decimation as a function of old site types. The argument of $\mathcal{N}$ and $\overline{\mathcal{N}}$ is $N-1$ in order to give a total of $4^{N-1}$ sites.

\begin{tabular}{cccccccccr}
\hline \hline New & 1 & 2 & 3 & 4 & $5 . R 1$ & $5 . R 3$ & $6 . R 2$ & $6 . R 4$ & Total \\
\hline 1 & $\mathcal{N}$ & $\mathcal{N}$ & $\mathcal{N}$ & $\mathcal{N}$ & 0 & 0 & 0 & 0 & $4 \mathcal{N}$ \\
2 & $\mathcal{N}$ & $\mathcal{N}$ & $\mathcal{N}$ & $\mathcal{N}$ & 0 & 0 & 0 & 0 & $4 \mathcal{N}$ \\
3 & 0 & $\mathcal{N}$ & $\mathcal{N}$ & 0 & $\mathcal{N}$ & 0 & 0 & $\mathcal{N}$ & $4 \mathcal{N}$ \\
4 & $\mathcal{N}$ & 0 & 0 & $\mathcal{N}$ & 0 & $\mathcal{N}$ & $\mathcal{N}$ & 0 & $4 \mathcal{N}$ \\
$5 . R 1$ & 0 & $\bar{N}$ & $\bar{N}$ & 0 & $\overline{\mathcal{N}}$ & 0 & 0 & $\overline{\mathcal{N}}$ & $4 \overline{\mathcal{N}}$ \\
$5 . R 3$ & 0 & $\overline{\mathcal{N}}$ & $\overline{\mathcal{N}}$ & 0 & $\overline{\mathcal{N}}$ & 0 & 0 & $\overline{\mathcal{N}}$ \\
$6 . R 2$ & $\overline{\mathcal{N}}$ & 0 & 0 & $\overline{\mathcal{N}}$ & 0 & $\overline{\mathcal{N}}$ & $\overline{\mathcal{N}}$ & 0 & $4 \overline{\mathcal{N}}$ \\
$6 . R 4$ & $\overline{\mathcal{N}}$ & 0 & 0 & $\overline{\mathcal{N}}$ & 0 & $\overline{\mathcal{N}}$ & $\overline{\mathcal{N}}$ \\
\hline \hline
\end{tabular}

$$
\begin{aligned}
D= & \left(1-d_{i(p-1)} e_{i(p-2)}-d_{i(p-2)} e_{i(p-3)}\right)\left(1-d_{i(p+3)} e_{i(p+2)}-d_{i(p+2)} e_{i(p+1)}\right) \\
& -\left(1-d_{i(p-1)} e_{i(p-2)}-d_{i(p-2)} e_{i(p-3)}\right)\left(1-d_{i(p+3)} e_{i(p+2)}\right) d_{i(p+1)} e_{i(p)} \\
& -\left(1-d_{i(p+3)} e_{i(p+2)}-d_{i(p+2)} e_{i(p+1)}\right)\left(1-d_{i(p-2)} e_{i(p-3)}\right) d_{i(p)} e_{i(p-1)}
\end{aligned}
$$

and the new type $i^{\prime}(p)$ of the preserved site is determined by the criteria introduced above.

The recursion relations (15) can be applied only $N-1$ times. After this number of renormalizations the $N$ th periodic approximation system becomes a linear chain where all coefficients to the right and left, respectively, are equal. Thus, from that point on renormalization is much easier and boils down to the following simple rules for the three remaining parameters $e, d$, and $g:^{7}$

$$
e^{\prime}=\frac{e^{2}}{1-2 e d}, \quad d^{\prime}=\frac{d^{2}}{1-2 e d}, \quad g^{\prime}=\frac{g}{1-2 e d} .
$$

\section{B. Even sites}

As we have indicated in the Introduction, some extra difficulties arise when we try to evaluate the contributions of the even-numbered sites to the physical properties of the system: Certain even sites do not constitute nodes and allow left and right (odd) neighbors to interact without their interference. This turns out to complicate considerably the derivation of recursion relations analogous to those in Eq. (15), which are valid for all (odd) sites.

Therefore, a new strategy has to be chosen. Without loss of generality we may focus on the sites with labels $4 t$, $t \in \mathbb{Z}$, which are the right neighbors of the odd sites $4 t-1$ discussed in Sec. III A. The basic idea is to calculate any desired diagonal Green's coefficient $G_{4 \hat{t}, 4 \hat{t}}^{(N)}$ via the above renormalization scheme for the odd sites by keeping an accompanying extra equation, which links the even "ghost" site to the adjacent pivotal odd site $4 \hat{t}-1$. This equation has to be renormalized as well in each decimation step. After many iterations of the renormalization procedure, the site $4 \hat{t}-1$ will eventually be decoupled from all its odd neighbors, but not from its "true" neighbor $4 \hat{t}$. At this point $G_{4 \hat{t}, 4 \hat{t}}^{(N)}$ can easily be derived from the limit form of the extra equation.

Let us restart from Eq. (3) with $m=4 \hat{t}$. In order to simplify the notation, we will again suppress this second index as well as the approximation order symbol $N$. If we keep in the first step decimation the sites numbered as $4 t-1, t \in \mathbb{Z}$, then the diluted system looks much like the one we would obtain for the choice $m=4 \hat{t}-1$. The only differences concern the shape of the equations for $n=4 \hat{t}-1$ and $n=4 \hat{t}+3$, which become

$$
\begin{aligned}
& G_{4 \hat{\imath}-1}=\hat{g}_{4 \hat{\imath}-1}+d_{4 \hat{\imath}-1} G_{4 \hat{\imath}-5}+e_{4 \hat{\imath}-1} G_{4 \hat{\imath}+3}, \\
& G_{4 \hat{\imath}+3}=\widetilde{g}_{4 \hat{\imath}+3}+d_{4 \hat{\imath}+3} G_{4 \hat{\imath}-1}+e_{4 \hat{\imath}+3} G_{4 \hat{t}+7} .
\end{aligned}
$$

In addition, we have to take along the equation connecting $G_{4 \hat{t}}$, since this is the quantity which we are interested in. Its general form is

$$
G_{4 \hat{t}}=\bar{g}_{4 \hat{\imath}}+\delta_{4 \hat{t}} G_{4 \hat{\imath}-1}+\varepsilon_{4 \hat{t}} G_{4 \hat{t}+3} .
$$

In Eqs. (18) and (19) we have introduced new inhomogeneities $\hat{g}, \tilde{g}, \bar{g}$ and new interaction coefficients $\delta, \varepsilon$, which also depend on the type of the pivotal site $4 \hat{t}-1$. Therefore, we have to handle the additional parameter set $\left\{\hat{g}_{i}, \widetilde{g}_{i}, \bar{g}_{i}, \delta_{i}, \varepsilon_{i} \mid i=1,2,3,4,5,6\right\}$ that will be renormalized in each decimation step. A careful analysis yields the explicit expressions for these parameters as functions of the site types, as summarized in Table IV. We emphasize that the old coefficients involved, namely $d_{i}$ and $e_{i}$, do not have to be modified.

Regarding the general renormalization operation, we can proceed along the same lines as described in Sec. III A. However, the string of Eqs. (14) must be replaced by the following enlarged system: 
TABLE IV. Renormalized interaction coefficients required for evaluating the contributions of the even sites to the DS. The classification is induced by the odd-site type scheme. The quantity $D_{3}$ is given by $D_{3}=1-2 x^{2}$.

\begin{tabular}{cccccc}
\hline \hline $\begin{array}{c}\text { Site } \\
\text { type }\end{array}$ & $\hat{g}_{i}$ & $\widetilde{g}_{i}$ & Coefficients & & $\delta_{i}$ \\
\hline 1 & $g x\left(1-x^{2}\right) / D_{1}$ & $g x\left(x^{2}+x_{l}\right) / D_{1}$ & $g\left(1-x^{2}\right) / D_{3}$ & $x\left(1-x^{2}\right) / D_{3}$ & $\varepsilon_{i}$ \\
2 & $g x\left(1-x^{2}\right) / D_{1}$ & $g x\left(x^{2}+x_{l}\right) / D_{2}$ & $g\left(1-x^{2}\right) / D_{3}$ & $x\left(1-x^{2}\right) / D_{3}$ & $x\left(x^{2}+x_{l}\right) / D_{3}$ \\
3 & $g x\left(1-x^{2}+x_{l}\right) / D_{2}$ & $g x\left(x^{2}+x_{l}\right) / D_{1}$ & $g\left(1-x^{2}\right) / D_{3}$ & $x\left(1-x^{2}+x_{l}\right) / D_{3}$ & $x\left(x^{2}+x_{l}\right) / D_{3}$ \\
4 & $g x\left(1-x^{2}\right) / D_{1}$ & $g x\left(x^{2}+x_{l}\right) / D_{1}$ & $g\left(1-x^{2}\right) / D_{3}$ & $x\left(1-x^{2}\right) / D_{3}$ & $x\left(x^{2}+x_{l}\right) / D_{3}$ \\
5 & $g x\left(1-x^{2}+x_{l}\right) / D_{2}$ & $g x\left(x^{2}+x_{l}\right) / D_{1}$ & $g\left(1-x^{2}\right) / D_{3}$ & $x\left(1-x^{2}+x_{l}\right) / D_{3}$ & $x\left(x^{2}+x_{l}\right) / D_{3}$ \\
6 & $g x\left(1-x^{2}\right) / D_{1}$ & $g x\left(x^{2}+x_{l}\right) / D_{2}$ & $g\left(1-x^{2}\right) / D_{3}$ & $x\left(1-x^{2}\right) / D_{3}$ & $x\left(x^{2}+x_{l}\right) / D_{3}$ \\
\hline \hline
\end{tabular}

$$
\begin{aligned}
& G_{p-3}=d_{i(p-3)} G_{p-4}+e_{i(p-3)} G_{p-2}, \\
& G_{p-2}=d_{i(p-2)} G_{p-3}+e_{i(p-2)} G_{p-1}, \\
& G_{p-1}=d_{i(p-1)} G_{p-2}+e_{i(p-1)} G_{p}, \\
& G_{p}=d_{i(p)} G_{p-1}+e_{i(p)} G_{p+1}+\hat{g}_{i(p)}, \\
& G_{p+1}=d_{i(p+1)} G_{p}+e_{i(p+1)} G_{p+2}+\widetilde{g}_{i(p+1)}, \\
& G_{p+2}=d_{i(p+2)} G_{p+1}+e_{i(p+2)} G_{p+3}, \\
& G_{p+3}=d_{i(p+3)} G_{p+2}+e_{i(p+3)} G_{p+4}, \\
& G_{p+4}=d_{i(p+4)} G_{p+3}+e_{i(p+4)} G_{p+5}, \\
& G_{p+5}=d_{i(p+5)} G_{p+4}+e_{i(p+5)} G_{p+6}, \\
& G_{p+6}=d_{i(p+6)} G_{p+5}+e_{i(p+6)} G_{p+7}, \\
& G_{p+7}=d_{i(p+7)} G_{p+6}+e_{i(p+7)} G_{p+8} .
\end{aligned}
$$

$\hat{g}_{i^{\prime}(p)}^{\prime}=\frac{1}{D}\left[\hat{g}_{i(p)}\left(1-e_{i(p+1)} d_{i(p+2)}-e_{i(p+2)} d_{i(p+3)}\right)+\widetilde{g}_{i(p+1)}\left(1-e_{i(p+2)} d_{i(p+3)}\right) e_{i(p)}\right]\left(1-e_{i(p-3)} d_{i(p-2)}-e_{i(p-2)} d_{i(p-1)}\right)$,

$\widetilde{g}_{i^{\prime}(p+1)}^{\prime}=\frac{1}{F} \widetilde{g}_{i(p+1)} d_{i(p+2)} d_{i(p+3)} d_{i(p+4)}\left(1-e_{i(p+5)} d_{i(p+6)}-e_{i(p+6)} d_{i(p+7)}\right)$.

Note that the interaction coefficients $d_{i}, e_{i}$ are renormalized in the way already described above [see Eq. (15)] and that the quantity $D$ is given by Eq. (16). $F$, on the other hand, is defined by

$$
\begin{aligned}
F= & \left(1-e_{i(p+1)} d_{i(p+2)}-e_{i(p+2)} d_{i(p+3)}\right)\left(1-e_{i(p+5)} d_{i(p+6)}-e_{i(p+6)} d_{i(p+7)}\right) \\
& -\left(1-e_{i(p+1)} d_{i(p+2)}-e_{i(p+2)} d_{i(p+3)}\right)\left(1-e_{i(p+6)} d_{i(p+7)}\right) e_{i(p+4)} d_{i(p+5)} \\
& -\left(1-e_{i(p+5)} d_{i(p+6)}-e_{i(p+6)} d_{i(p+7)}\right)\left(1-e_{i(p+1)} d_{i(p+2)}\right) e_{i(p+3)} d_{i(p+4)} .
\end{aligned}
$$

Finally, from Eq. (21) we get

$$
\begin{aligned}
& \bar{g}_{i^{\prime}(4 \hat{\imath})}^{\prime}=\bar{g}_{i(4 \hat{\imath})}+\frac{1}{H} \varepsilon_{i(4 \hat{t})} \widetilde{g}_{i(p+1)}\left(1-e_{i(p+2)} d_{i(p+3)}\right), \\
& \delta_{i^{\prime}(4 \hat{t})}^{\prime}=\delta_{i(4 \hat{\imath})}+\frac{1}{H} \varepsilon_{i(4 \hat{t})} d_{i(p+1)}\left(1-e_{i(p+2)} d_{i(p+3)}\right), \\
& \varepsilon_{i^{\prime}(4 \hat{t})}^{\prime}=\frac{1}{H} \varepsilon_{i(4 \hat{t})} e_{i(p+1)} e_{i(p+2)} e_{i(p+3)} .
\end{aligned}
$$

The quantity $H$ is given by

$H=1-e_{i(p+1)} d_{i(p+2)}-e_{i(p+2)} d_{i(p+3)}$.
If we deal with a system representing the $N$ th-order periodic approximation to the fractal limit, then the recursion relations have to be used a finite number of times until a renormalized linear chain has emerged. After that the further decimation is again realized by simpler recursion relations:

$$
\begin{aligned}
& \hat{g}^{\prime}=\frac{\hat{g}+e \widetilde{g}}{1-2 d e}, \quad \widetilde{g}^{\prime}=\frac{d \widetilde{g}}{1-2 d e}, \quad \bar{g}^{\prime}=\bar{g}+\varepsilon \widetilde{g}, \\
& \delta^{\prime}=\delta+\varepsilon d, \quad \varepsilon^{\prime}=\varepsilon e .
\end{aligned}
$$




\section{Exact renormalization in the fractal limit}

In Secs. III A and III B we have worked out in a constructive way the Green's-function renormalization scheme for $N$ th-order periodic approximations to the Koch terragon. We chose this specific way of presentation in order to make clear the basic ideas and various subtle difficulties that might be glossed over by a more deductive treatment. The price to be paid is a certain lack of transparency. We are now, however, in a position to look back on what we have accomplished and to summarize concisely the renormalization principles involved. This is particularly satisfying if we focus, from the outset, on the fractal limit $(N \rightarrow \infty)$, which we are mainly interested in anyway.

The results of the next section will confirm that, in fact, the contributions of all sites $n \in \mathbb{Z}$ have to be taken into account for deriving the relevant physical properties of the system. Yet we may restrict our summation to the sites labeled as $4 t-1, t \in \mathbb{Z}$ : The original sites numbered as $4 t, 4 t+1,4 t+2$ quite generally can be covered by attaching them to the $4 t-1$ sites via finite sets of ghost equations, as discussed in Sec. III B. Moreover, the sites with labels $4 t+1$ are directly related to those numbered as $4 t-1$ through the symmetry operation $n \mapsto-n$. The pertinent calculations are straightforward in each case and need not be discussed here in detail.

After the initial decimation step discussed above [Eq. (7)], only the original $4 t-1$ sites are retained and the fractal is represented by an infinite sequence, which is composed of the site type figures $\{1,2,3,4,5,6\}$ linked by the bond parameters $d_{i}, e_{i}$ and decorated with the on-site parameters $g_{i}$. The following short segment centered around the original site with label -1 may serve to illustrate this:

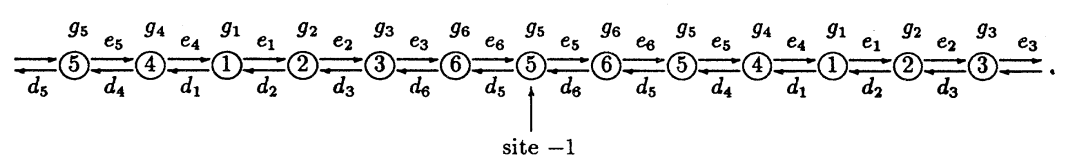

The complete sequence can be generated in an exponentially fast way from the "seed symbol" 1 through the inflation rules

$$
\begin{aligned}
& 1 \rightarrow 2341, \quad 2 \rightarrow 2341, \quad 3 \rightarrow 2365, \\
& 4 \rightarrow 6541, \quad 5 \rightarrow 2365, \quad 6 \rightarrow 6541 .
\end{aligned}
$$

Our main task is to calculate the diagonal Green's coefficient associated with any given site $4 t-1$. This is most efficiently achieved by repeated site decimation and renormalization of the on-site parameter $g$, which ultimately becomes equal to the coefficient desired. But note that decimation here does not simply mean the inversion of the inflation rules: Depending on the site in question that obviously has to be preserved in each step, a choice between four sublattices has to be made. As a consequence, other four-strings than those covered n (31) must also be dealt with.

Our job consists of two parts: (i) determination of the new types of the preserved sites, (ii) renormalization of the parameters $d_{i}, e_{i}$ and $g_{i}$. The first part could be carried out using Table II. This tableau, however, is somewhat unwieldy, contains additional information not needed here, and does not provide immediate unique type assignment. Yet we can extract from it an unambiguous set of rules with the aid of consistency considerations. These simple rules involve only neighboring sites on the preserved sublattice and are displayed in Table V.

The table actually accounts for the quite unusual and sophisticated renormalization scheme that has to be applied to our infinite sequence of figures: At each stage of the decimation there are four distinct sublattices com- posed of the site types $\{1,5\},\{2,6\},\{3,5\}$, and $\{4,6\}$, respectively. The preserved site, whose contribution we are interested in, has to reside in one of these sublattices, but its membership perpetually changes in the course of the decimation. The current status determines the specific set of decimation rules that has to be chosen. Each set contains six individual rules, as six different site types have to be generated to build up the new sequence. The four decimation types directly correspond to the majority rules $R 1-R 4$, defined in (12).

We emphasize here that the infinite-site-type sequence associated with our fractal is simultaneously exactly invariant with respect to all four decimation schemes. This situation is quite different from the one discussed in familiar examples of exactly renormalizable aperiodic lattices: In those investigations individual sites, except (perhaps) a central one, do not matter, and a unique decimation procedure eliminates all but the sites on a hierarchical skeleton. By way of contrast, we are dealing with a rich, multiply renormalizable self-similar structure, which allows us to cover each individual site.

The ultimate realization of the $\mathrm{RG}$ consists in the renormalization of the group parameters $d_{i}, e_{i}$, and $g_{i}$. Ordinary singular renormalizability would guarantee that the same transformation rules apply at each stage of the process. The multiple renormalizability of our system, however, induces a more intricate scheme reminiscent of the iterated-function-systems approach to fractal image compression by Barnsley: ${ }^{20}$ Associated with each fixed preserved site $4 t-1$ is a well-defined sequence $\left\{r_{s} \mid s \in \mathbb{N}\right\}$ composed of the numbers $\{1,2,3,4\}$, which prescribes the correct succession of the four possible different blocks of 
renormalization equations to be employed. Each distinct parameter renormalization scheme consists of 48 equations and can be distilled from the general transformation (15) by picking the appropriate decimation type as a function of the current sublattice accommodating the site in question. The sequence $\left\{r_{s}\right\}$ is clearly related to the 4adic representation of $t$.

Case (a) $t \leq 0$ : Let $\bar{t}=-t$ and decompose this number as

$$
\bar{t}=\sum_{s \in \mathbb{N}} \alpha_{s} 4^{s-1}
$$

where $\alpha_{s} \in\{0,1,2,3\}$. By inspection we can find immediately the mapping $r_{s}=f\left(\alpha_{s}\right)$, which is specified by

$$
0 \mapsto 1, \quad 1 \mapsto 4, \quad 2 \mapsto 3, \quad 3 \mapsto 2
$$

Case (b) $t>0$ : Let $\bar{t}=t-1$ and represent this number as

$$
\bar{t}=\sum_{s \in \mathbb{N}} \beta_{s} 4^{s-1},
$$

with $\beta_{s} \in\{0,1,2,3\}$. Here we get $r_{s}=g\left(\beta_{s}\right)$, where the mapping $g$ is defined as

$$
0 \mapsto 2, \quad 1 \mapsto 3, \quad 2 \mapsto 4, \quad 3 \mapsto 1 .
$$

In order to give an example, let us assume that site
$4 t-1=-121$ is the one to be retained. It belongs to case (a), so

$$
\bar{t}=30-2 \times 4^{0}+3 \times 4^{1}+1 \times 4^{2},
$$

and

$$
\begin{aligned}
& \alpha_{1}=2, \quad \alpha_{2}=3, \quad \alpha_{3}=1, \\
& \alpha_{s}=0 \text { for all } s \geq 4 .
\end{aligned}
$$

This implies the following sequence of site-type and parameter renormalization schemes: step 1, type 3; step 2, type 2; step 3, type 4; all further steps, type 1 .

We are faced here with a quite unfamiliar RG, which may deserve the name "group" more than many wellknown examples: It is generated by four nonequivalent operations that have to be composed according to the rules just described. Each preserved site has its individual and unique compositional sequence. But note that for finite $t$ all these sequences end in uniform tails, consisting of 1 's when $t<0$ and of 2's when $t \geq 0$. This corresponds to the fact that all sites within a finite distance from the center are finally drawn into it in the course of the de-

\begin{tabular}{|c|c|c|c|c|c|c|}
\hline $\begin{array}{l}\text { Deci- } \\
\text { mation } \\
\text { type } \\
\end{array}$ & $\begin{array}{l}\text { Next-nearest } \\
\text { preserved } \\
\text { neighbor } \\
\text { to the left }\end{array}$ & $\begin{array}{c}\text { Nearest } \\
\text { preserved } \\
\text { neighbor } \\
\text { to the left }\end{array}$ & $\begin{array}{l}\text { Preserved } \\
\text { site in } \\
\text { question }\end{array}$ & $\begin{array}{l}\text { Nearest } \\
\text { preserved } \\
\text { neighbor } \\
\text { to the right }\end{array}$ & $\begin{array}{l}\text { Next-nearest } \\
\text { preserved } \\
\text { neighbor } \\
\text { to the right }\end{array}$ & $\begin{array}{l}\text { New } \\
\text { site } \\
\text { type }\end{array}$ \\
\hline \multirow[t]{6}{*}{1} & & 1 & 1 & 1 & & 1 \\
\hline & & 1 & 1 & 5 & & 2 \\
\hline & 1 & 1 & 5 & 1 & & 3 \\
\hline & & 5 & 1 & 1 & & 4 \\
\hline & 5 & 1 & 5 & 1 & & 5 \\
\hline & & 5 & 1 & 5 & & 6 \\
\hline \multirow[t]{6}{*}{2} & & 6 & 2 & 2 & & 1 \\
\hline & & 2 & 2 & 2 & & 2 \\
\hline & & 2 & 2 & 6 & & 3 \\
\hline & & 2 & 6 & 2 & 2 & 4 \\
\hline & & 6 & 2 & 6 & & 5 \\
\hline & & 2 & 6 & 2 & 6 & 6 \\
\hline \multirow[t]{6}{*}{3} & & 5 & 3 & 3 & & 1 \\
\hline & & 3 & 3 & 3 & & 2 \\
\hline & & 3 & 3 & 5 & & 3 \\
\hline & & 3 & 5 & 3 & 3 & 4 \\
\hline & & 5 & 3 & 5 & & 5 \\
\hline & & 3 & 5 & 3 & 5 & 6 \\
\hline \multirow[t]{6}{*}{4} & & 4 & 4 & 4 & & 1 \\
\hline & & 4 & 4 & 6 & & 2 \\
\hline & 4 & 4 & 6 & 4 & & 3 \\
\hline & & 6 & 4 & 4 & & 4 \\
\hline & 6 & 4 & 6 & 4 & & 5 \\
\hline & & 6 & 4 & 6 & & 6 \\
\hline
\end{tabular}
cimation and assume site types 5 and 6 , respectively.

\section{NUMERICAL RESULTS}

We now present several results which were obtained using the $R G$ method realized by the recursion relations

TABLE V. New type of any preserved site after decimation as a unique function of old type of the site itself and the preserved nearest and next-nearest neighbors. 
derived above. The major goal is to present the DS as a function of the energy and the model parameters. The presentation of the results is best achieved by a series of illustrations wherein we seek to lay stress upon some important features of both the method and the model.

For a first check of our results we compare them with the exact analytical expression for the DS when $N=1$. The latter is most easily obtained from the Schrödinger equation defined by the Hamiltonian (1) by exerting the first decimation step as discussed in Sec. II: This results in the Schrödinger equation for a "pure" linear chain with unit period and interaction, but renormalized energy given by

$$
E^{\prime}=\frac{E^{4}-4 E^{2}+2-2 \lambda E-\lambda^{2} E^{2}}{1+\lambda E}
$$

Now the integrated density of states $\kappa$ of an unperturbed linear chain is a well-known quantity. Observing that the scale transformation associated with the decimation implies $\kappa \rightarrow 4 \kappa$ we immediately obtain

$\kappa_{1}(E)=\frac{1}{4 \pi} \cos ^{-1}\left(\frac{E^{4}-4 E^{2}+2-2 \lambda E-\lambda^{2} E^{2}}{1+\lambda E}\right)$.

By simply differentiating this expression we get the DS as

$$
\rho_{1}(E)=\frac{1}{4 \pi} \frac{\left(E^{2}-2\right)\left[4 E(1+\lambda E)-\lambda\left(E^{2}-2\right)\right]-\lambda^{2} E(2+\lambda E)}{(1+\lambda E)\left\{\left(E^{2}-2\right)\left[\left(4-E^{2}\right) E^{2}+4 \lambda E+2 \lambda^{2} E^{2}\right]-\lambda^{2} E^{2}(2+\lambda E)^{2}\right\}^{1 / 2}}
$$

This analytical result is plotted in Fig. 2(a) for $\lambda=0.1$. For comparison we have calculated $\rho_{1}(E)$ also by the Green's-function technique described above. If the contributions of all sites are taken into account and $\epsilon$ in $G_{n n}^{(1)}(E+i \epsilon)$ is chosen sufficiently small $\left(\epsilon \leq 10^{-4}\right)$, then we end up with practically the same graph. Its prominent feature is the splitting of the true chain spectrum in four clustered subbands.

Figures 2(b) and 2(c) distill the contributions of particular sites to the DS. These pictures clearly demonstrate that the gap structure is identical for different site classes, yet these classes contribute very differently to the nonvanishing part of $\rho_{1}$. This is most dramatically felt in the neighborhood of the band edges. So, in order to get the full information on the system, all sites have to be considered.

In Fig. 3 we depict the DS for the approximation levels $N=2$ and 3, respectively, but for the same value of $\lambda$ as chosen for Fig. 2. The perpetual process of subband splitting as derived in Ref. 15 is clearly recovered. Since the number of minibands grows exponentially with $N$, the shape of the DS already bears very little resemblance to that of the simple linear chain.

Figure 4 demonstrates that the Green's-function approach is also capable of revealing the local scaling behaviof of the (integrated) density of states, which has been proven rigorously in Ref. 15. The graph of $\kappa$ vs $E$ scales, for instance, in the neighborhood of the point $(E, \kappa)=\left(1, \frac{3}{10}\right)$ according to the following law:

$$
\kappa(1+\Delta E)-\frac{3}{10}=\frac{1}{16}\left[\kappa\left(1+v_{\max }^{2} \Delta E\right)-\frac{3}{10}\right] .
$$

Here $v_{\max }^{2}=23.42 \ldots$ is a critical exponent which can be extracted from the exact transfer matrix RG (see Ref. 15 for details). Equation (31) implies the following local scaling law for the DS $\rho$ :

$$
\rho(1+\Delta E)=\frac{v_{\max }^{2}}{16} \rho\left(1+v_{\max }^{2} \Delta E\right) .
$$

This relation is confirmed by the Green's-function technique introduced in this paper. In principle, scale invariance is only achieved when the precise form of $\rho$ is considered, taking into account the contributions of all sites. However, since the number of sites to be gathered increases very fast, we have checked whether dilational symmetry shows up already in the contributions of particular subsequences of sites. This is indeed the case: For the simplest sequence (where the sites are of type 1 after any decimation step) Figs. 4(a) -4(d) clearly reflect the expected scaling behavior. The depicted component of the local DS rapidly converges to a universal function when the rescaling factors defined by Eq. (41) are used.

In Fig. 5 we inspect the superlocalized quantum states present in our system for special values of the crosshopping strength $\lambda$. The existence of such "molecular states" in a quasi-one-dimensional lattice was discovered in Ref. 15. In Fig. 5(a) the complete DS for $\lambda=1$ and $N=2$ is shown. Figure $5(\mathrm{~b})$ depicts precisely the same quantity, but now the scale of the vertical axis has been reduced by the factor $10^{-3}$. This is a good way to get an idea of the different orders of magnitude needed to describe the height of $\rho_{2}$ at the superlocalized energy level $(E=1)$ and at the ordinary niveaux.

In Figs. 5(c) and 5(d) we depict contributions to $\rho_{2}$ of individual classes of sites. We notice that at least one of these classes does not contribute at all to the superlocalized state peak [Fig. 5(d)].

We emphasize that all the figures presented and discussed in this section are meant to illustrate the power of the Green's-function approach to work out the most characteristic aspects of electron dynamics on the Koch curve. These aspects, which have first been detected using the TMM, are simultaneously the most unusual ones regarding the plethora of now existing deterministic nonperiodic models.

The more common features of our system can also be correctly dealt with by the Green's-function RG. All this demonstrates that such a technique is a valid and efficient tool for the investigation of deterministically disordered solids like fractals. 

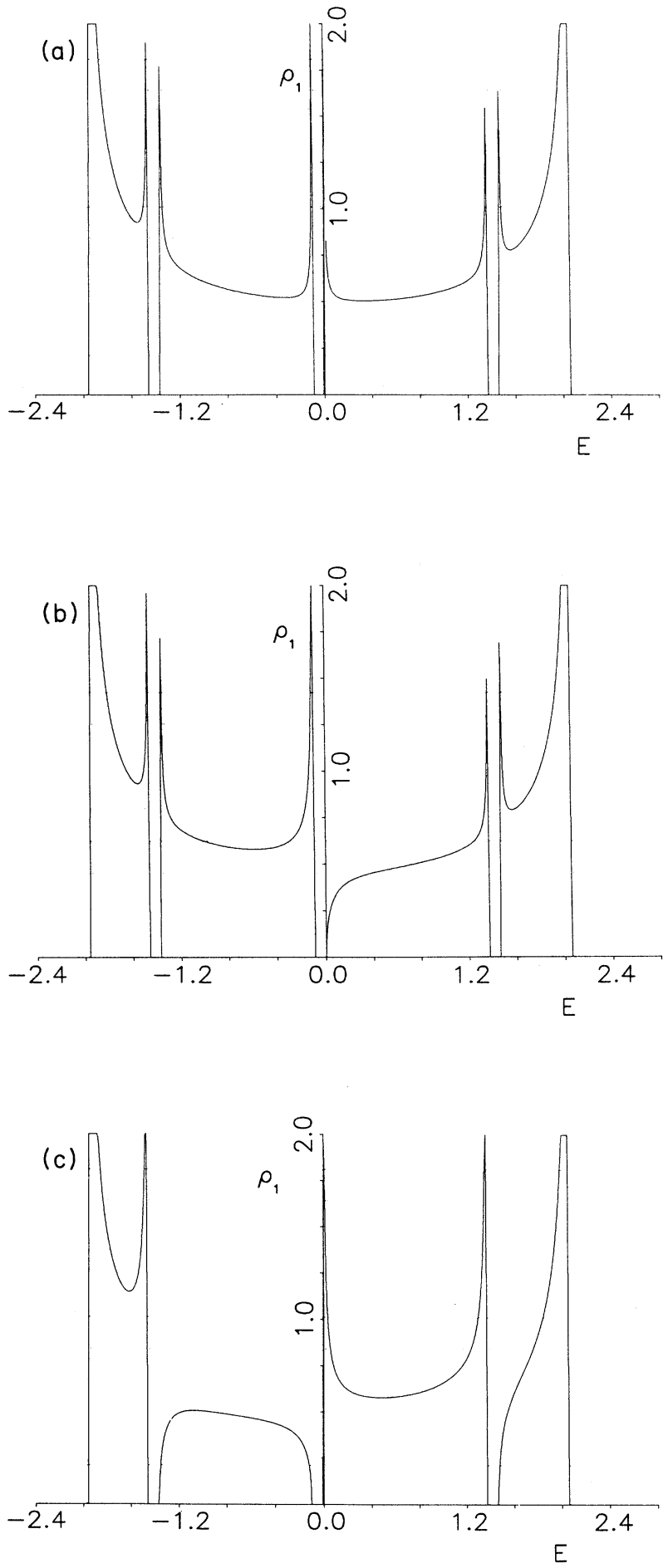

FIG. 2. Density of states as obtained in the first periodic approximation $(N=1)$ for cross-hopping strength $\lambda=0.1$. (a) Sum of contributions of all four different site types. (b) Contributions of sites $4 n-1, n \in \mathbb{Z}$. (c) Contributions of sites $4 n$, $n \in \mathbb{Z}$. Note that all DS shown in this figure and the following ones have been normalized by dividing by the number of sites involved [see Eq. (4)]. Therefore the contributions of subclasses of sites may appear larger than expected.
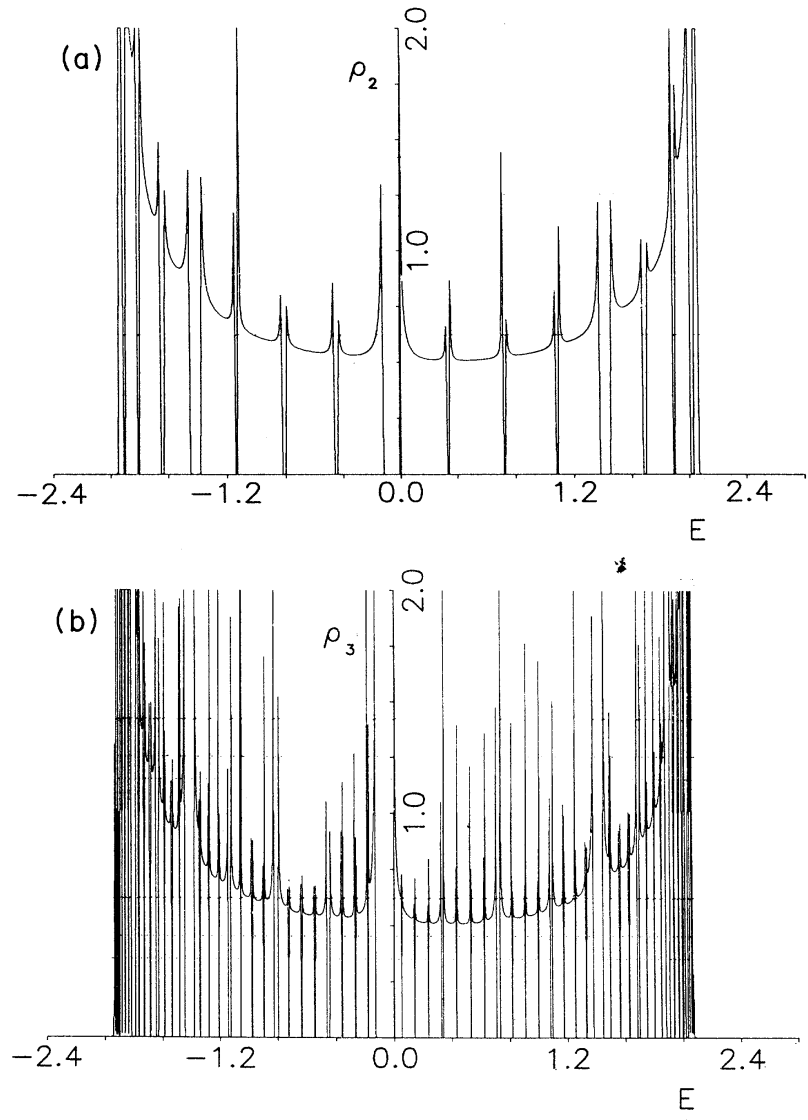

FIG. 3. Complete DS for $\lambda=0.1$ as in Fig. 2, but (a) secondorder approximation $(N=2)$; (b) third-order approximation $(N=3)$.

\section{CONCLUSIONS AND OUTLOOK}

In this paper we have explored a nontrivial tightbinding model on a fractal substrate by means of an exactly renormalizable Green's-function method. In order to implement the basic decimation idea a number of rather subtle aspects had to be taken into account. It turns out, in particular, that the following points are crucial for the evaluation of the DS via the diagonal Green'sfunction coefficients: (i) identification of all different types of sites, decimation rules, and bond renormalization schemes, (ii) identification of all possible decimation procedures as a function of site position on the fractal, (iii) gathering of the contributions of all sites.

These complications are a direct consequence of the lack of translational invariance in fractal systems and have to be mastered if exact results are desired. ${ }^{21}$ Our results are, in fact, in complete agreement with those derived using TMM in Ref. 15.

This finding indicates that Green's-function RG techniques should be useful methods for the treatment of other precisely or approximately self-similar model systems. ${ }^{22}$ Regarding further applications, we have to emphasize the necessity of observing points (ii) and (iii) above: Contributions of individual sites to the DS are 
certainly valuable quantities, as they give the correct gap positions and illustrate the band-splitting process. The restriction to one single contribution may feign, however, unphysical effects at the band edges; Fig. 2 illustrates that the complete DS exhibits singularities, while components associated with subsets of sites go smoothly to zero.

In the present paper we have used the renormalization scheme primarily for rapid numerical investigations. We have not yet exploited, however, the renormalization equations in order to derive analytical results concerning scaling relations and critical exponents. This is an interesting problem in its own right and is planned to be discussed elsewhere.

Let us also stress that the model structure investigated - the Koch terragon-is a nonbranching fractal. This topological property allows the transformation into a nonperiodic chain. Quite a few such deterministically disordered one-dimensional systems have been discussed in recent literature. But note that very few results are available for electron dynamics on branching fractals. We are confident that the methods and results obtained here pave the way for a systematic treatment of these more intricate objects. Work in this direction is in progress.
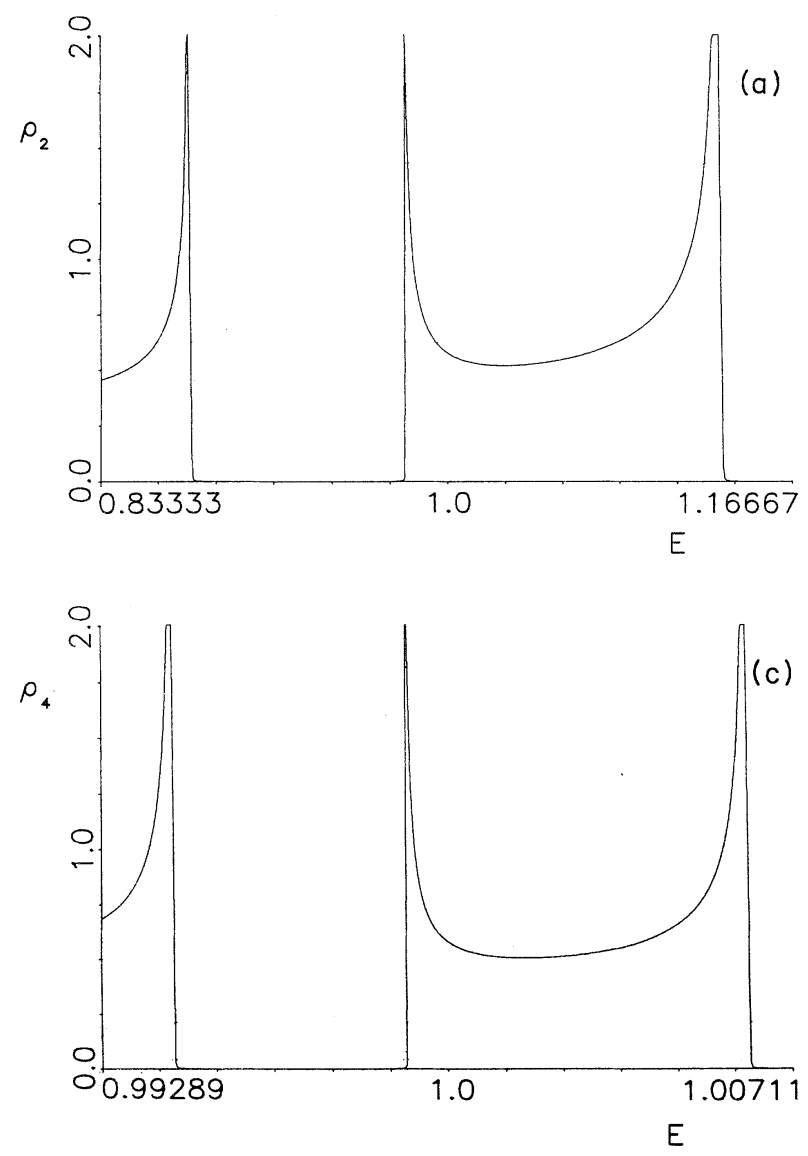

\section{ACKNOWLEDGMENTS}

The authors wish to thank CNPq (Brazil) and DAAD (Germany) for partial support of this work.

\section{APPENDIX A}

The initial decimation described by rule (7) transforms the fractal or its periodic approximations into a linear chain with hierarchically distributed single and double bonds. The associated Green's function is characterized by site-type dependent left and right interaction coefficients $d_{i}$ and $e_{i}$, respectively, and by renormalized site-type dependent inhomogeneities $g_{i}$. These expressions serve, in turn, as initial conditions for the subsequent general renormalization scheme (13), so we might call them $e_{i}^{(1)}, d_{i}^{(1)}$, and $g_{i}^{(1)}$. Their explicit form as presented in Table $I$ is easily derived by decimating Eq. (9).

To that end we may from the outset consider the aperiodic limit, which is governed by the Hamiltonian $H=\lim _{N \rightarrow \infty} H_{N}$ and its corresponding Green's function $G=(z-H)^{-1}$. Equation (9) then obtains the following form:
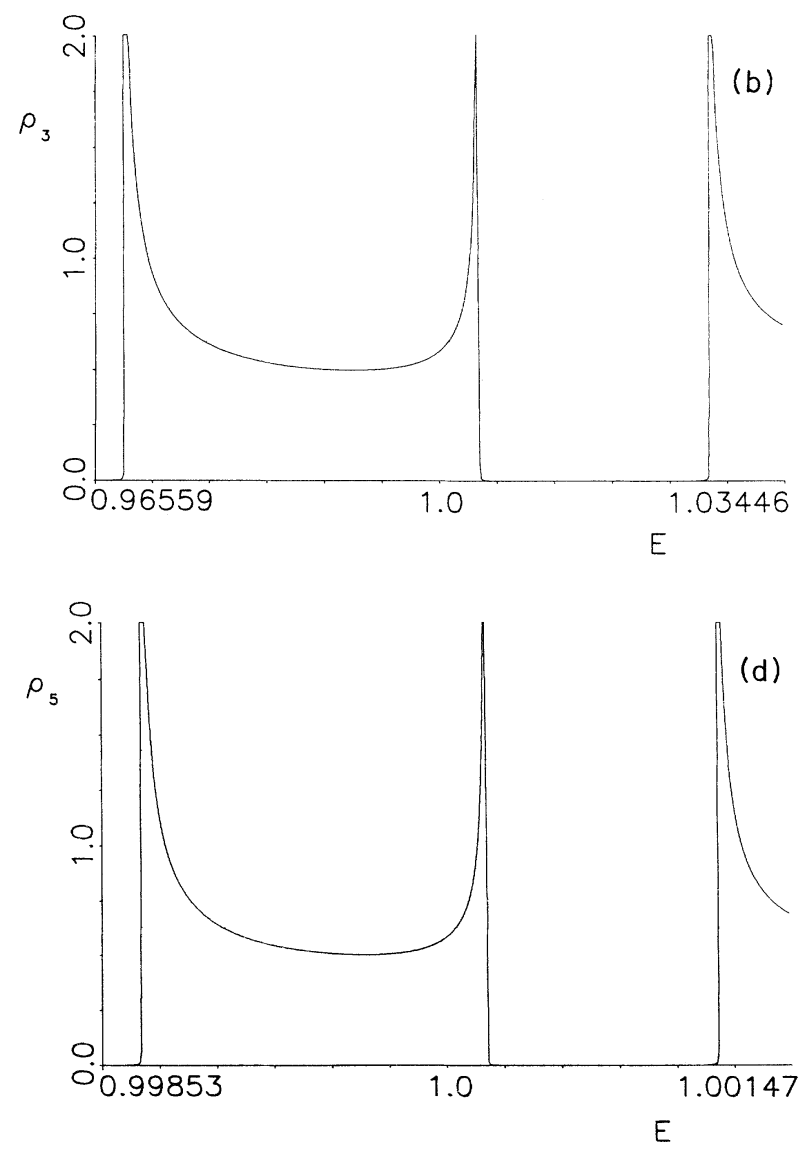

FIG. 4. Illustration of the local scaling behavior of $\rho(1+\Delta E)$ for $\lambda=3^{1 / 2}-1$. Only the contributions of the simplest siterenormalization class are taken into account. The self-affinity of the limit DS is simulated by a sequence of properly rescaled approximative DS. (a), (b), (c), and (d) correspond to approximation orders $N=2,3,4$, and 5, respectively. 


$$
\begin{aligned}
G_{n m}= & g \delta(n, m)+x_{l} t(n+1) G_{n+2, m}+x G_{n+1, m} \\
& +x G_{n-1, m}+x_{l} t(n-1) G_{n-2, m}
\end{aligned}
$$

where

$$
t(n)=\lim _{N \rightarrow \infty} t_{N}(n)=\delta(o, n)+\sum_{s \in N} \delta\left[\frac{1}{2} 4^{s}, n\left(\bmod 4^{s}\right)\right] .
$$

The initial decimation eliminates all sites except those labeled as $4 p-1, p \in \mathbb{Z}$. The decimated model is described by an infinite system of equations coupling the Green's coefficients $G_{4 p-1, m}, p \in \mathbb{Z}$. As far as the densities of states are concerned, we can restrict ourselves to the di- agonal case, i.e., $m=4 \hat{p}-1$, where $\hat{p}$ is some fixed integer. The renormalization procedure is formally concluded by the relabeling

$$
G_{4 p-1,4 \hat{p}-1} \rightarrow G_{p \hat{p}}
$$

In order to derive the desired expressions we start from the seven equations of type (A1), which are associated with $n=4 p-4,4 p-3,4 p-2,4 p-1,4 p, 4 p+1$, and $4 p+2$.

Eliminating from these equations first the even-site coefficients $\boldsymbol{G}_{4 p-4,4 \hat{p}-1}, \quad \boldsymbol{G}_{4 p-2,4 \hat{p}-1}, \quad \boldsymbol{G}_{4 p, 4 \hat{p}-1}, \quad$ and $\boldsymbol{G}_{4 p+2,4 \hat{p}-1}$, and then the odd-site coefficients $\boldsymbol{G}_{4 p-3,4 \hat{p}-1}$ and $G_{4 p+1,4 \hat{p}-1}$ we arrive at the following decimated equation:

$$
\begin{aligned}
G_{4 p-1,4 \hat{p}-1}= & \left(\frac{a_{4 p-1,4 \hat{p}-1}+a_{4 p+1,4 \hat{p}-1} b_{4 p}+a_{4 p-3,4 \hat{p}-1} b_{4 p-2}}{1-b_{4 p}^{2}-b_{4 p-2}^{2}}\right) \\
& +\left(\frac{b_{4 p} b_{4 p+2}}{1-b_{4 p}^{2}-b_{4 p-2}^{2}}\right) G_{4 p+3,4 \hat{p}-1}+\left(\frac{b_{4 p-4} b_{4 p-2}}{1-b_{4 p}^{2}-b_{4 p-2}^{2}}\right) G_{4 p-5,4 \hat{p}-1},
\end{aligned}
$$
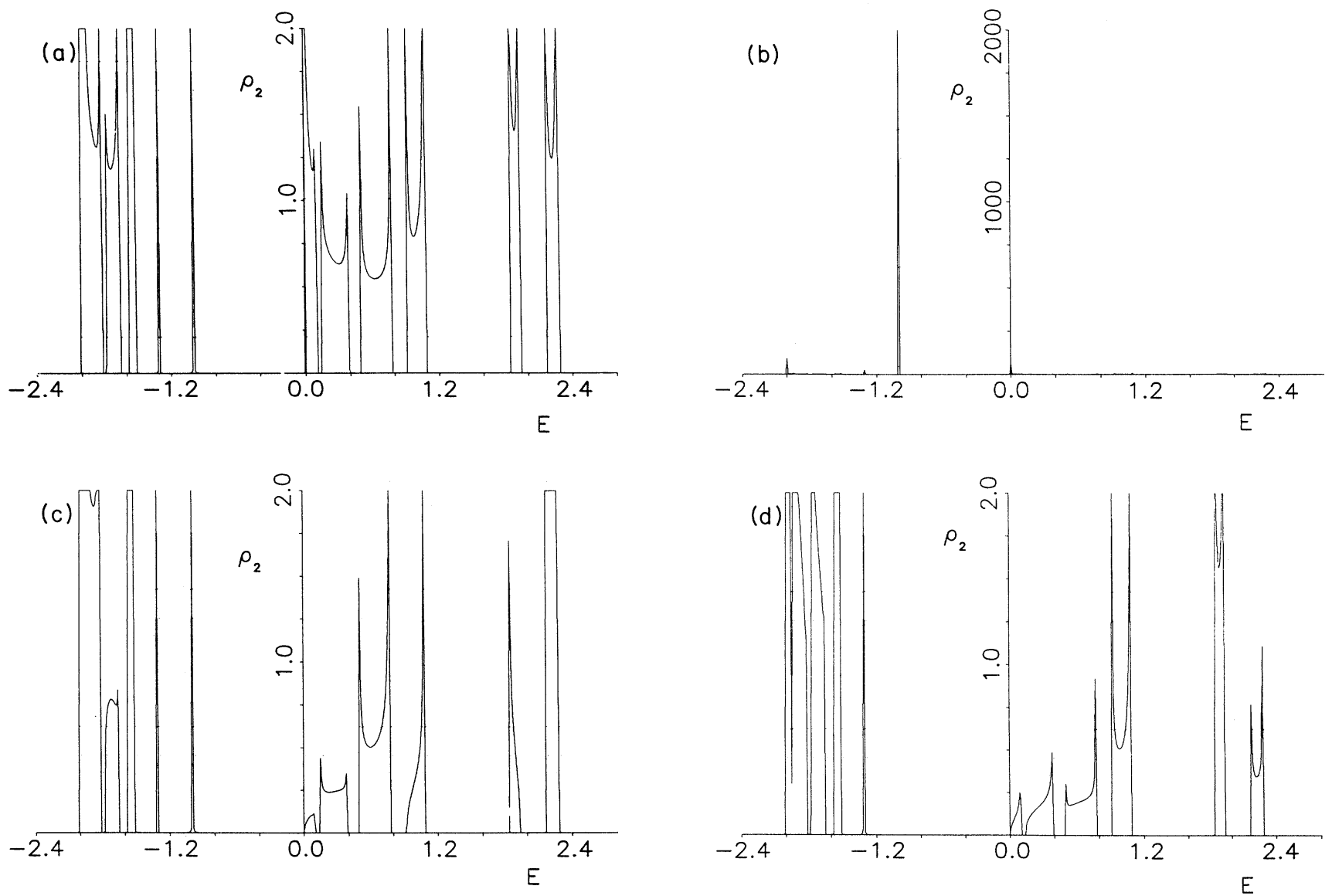

FIG. 5. Density of states for $\lambda=1$, where a superlocalized electronic phase exists at the energy level $E=-1$. The approximation order has been chosen as $N=2$. (a) and (b) Complete DS for different $\rho$ scales. (c) and (d) Partial DS as produced by subsets of sites of special type. 
where

$a_{q r}=\frac{g}{1-2 x^{2}}[\delta(q, r)+x \delta(q+1, r)+x \delta(q-1, r)]$

and

$$
b_{q}=\frac{x_{l} t(q)+x^{2}}{1-2 x^{2}} .
$$

Equation (A3) defines the renormalized inhomogeneity and the renormalized left and right interaction coefficients. These quantities depend on the local environment solely through the cross-hopping strengths $t(4 p-4), t(4 p-2), t(4 p)$, and $t(4 p+2)$.

Now (A2) immediately implies

$$
t(4 p-2)=t(4 p+2)=1 \text { for all } p \in \mathbb{Z} \text {. }
$$

We also observe that $t(4 p-4)$ differs from zero only when the sites $4 p-5$ and $4 p-1$ are coupled by a double bond. In a similar way, $t(4 p)$ only contributes when the sites $4 p-1$ and $4 p+3$ are connected by a double bond. Altogether we obtain the following table, which relates the relevant cross-hopping strengths to the type $i$ of site $4 p-1$ :

\begin{tabular}{ccccc} 
& $t(4 p-4)$ & $t(4 p-2)$ & $t(4 p)$ & $t(4 p+2)$ \\
\hline 1 & 0 & 1 & 0 & 1 \\
2 & 0 & 1 & 0 & 1 \\
3 & 0 & 1 & 1 & 1 \\
4 & 1 & 1 & 0 & 1 \\
5 & 0 & 1 & 1 & 1 \\
6 & 1 & 1 & 0 & 1
\end{tabular}

(A6)

Using this table the parameters $d_{i}, e_{i}$, and $g_{i}$ can be read off from Eq. (A3) immediately. Note that $g_{i}$ only appears in this equation when $p=\hat{p}$.

\section{APPENDIX B}

Table II prescribes how the new type of any given site surviving decimation can be inferred from the particular decimation rule employed and the local string of predecimation site types. We now illustrate how this prescription can be obtained.

As a matter of fact, the bond structure of the system prior to decimation, together with the majority rule, completely determines the site type after decimation. The lowest-order periodic approximation where the full identification scheme can be demonstrated belongs to $N=4$. In this case the unit cell consists of $4^{4}=256$ non- equivalent sites. The initial decimation as discussed in Appendix A reduces this unit cell to 64 sites linked by single and double bonds. The resulting string of bonds and site types looks like this:

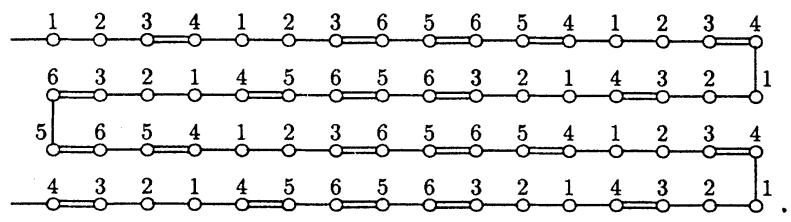

Here the first site on the third line, which is of type 5, corresponds to the site that bears the label $n=-1$ and is adjacent to the dilational center of the Koch curve.

We emphasize that the above sequence of single and double bonds is generated exclusively by rule (7), while type identification follows in a unique way from the classification scheme (11). The latter scheme can be used again to identify the site types in the renormalized bond structure obtained from (B1). But this new string of bonds is determined by the specific majority rule (12), which in turn depends on the pivotal site to be retained.

If we wish to preserve, for instance, the first site in (B1) and, as a consequence, the fifth, ninth, thirteenth, etc. site, then we have to resort to rule $R 1$. The decimation procedure produces the new structure

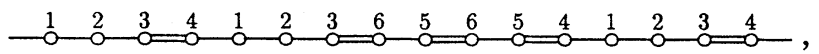

with renormalized single and double bonds and site types determined by scheme (11). By comparing (B1) to (B2) we realize, e.g., that the new site type 2 is obtained from an old site of type 1 constituting the center of the local type string 123412365. Accordingly, the latter string forms the entry on the second line (new type) of the first column (decimation rule) in Table II. The remaining entries in the same column can be obtained by inspecting appropriate other new sites.

Columns 2-4 of Table II can be determined in an analogous way by employing the majority rules $R 2, R 3$, and $R 4$, which preserve the second, third, and fourth site of $(B 1)$, respectively.
${ }^{1}$ F. J. Dyson, Phys. Rev. 92, 1331 (1953).

${ }^{2}$ P. W. Anderson, Phys. Rev. 109, 1492 (1958).

${ }^{3}$ P. Dean, Rev. Mod. Phys. 44, 127 (1972).

${ }^{4}$ R. J. Elliott, J. A. Krumhans1, and P. L. Leath, Rev. Mod. Phys. 46, 465 (1974).

${ }^{5}$ C. T. Papatriantafillou, E. N. Economou, and T. P. Eggarter,
Phys. Rev. B 13, 910 (1976).

${ }^{6}$ H. Aoki, J. Phys. C 13, 3369 (1980).

${ }^{7}$ C. E. T. Gonçalves da Silva and B. Koiller, Solid State Commun. 40, 215 (1981).

${ }^{8}$ For an introduction, see E. N. Economou, Green's Functions in Quantum Physics (Springer, Berlin, 1979), and references 
therein.

${ }^{9}$ One of the earliest investigations was performed by J. M. Langlois, A. M. S. Trembley, and B. W. Southern, Phys. Rev. B 28, 218 (1983).

${ }^{10}$ G. André and S. Aubry, Ann. Isr. Phys. Soc. 3, 133 (1980).

${ }^{11}$ J. Sokoloff and J. V. José, Phys. Rev. Lett. 49, 334 (1982).

${ }^{12}$ M. Kohmoto, L. P. Kadanoff, and C. Tang, Phys. Rev. Lett. 50, 1870 (1983); S. Ostlund, R. Pandit, D. Rand, H. J. Schellnhuber, and E. D. Siggia, ibid. 50, 1873 (1983).

13J. B. Sokoloff, Phys. Rep. 126, 189 (1985).

${ }^{14}$ For the more recent work on quasicrystalline models see $R$. B. Capaz, B. Koiller, and S. L. A. de Queiroz, Phys. Rev. B 42, 6402 (1990), and references therein.

${ }^{15}$ R. F. S. Andrade and H. J. Schellnhuber, Europhys. Lett. 10, 73 (1989).

16J. A. Ashraff and R. B. Stinchcombe, Phys. Rev. B 37, 5723 (1988).

${ }^{17}$ B. Simon, Adv. Appl. Math. 3, 463 (1982).
${ }^{18}$ B. B. Mandelbrot, The Fractal Geometry of Nature (Freeman, New York, 1983).

${ }^{19}$ Molecular states in higher-dimensional structures have been found, e.g., by R. Rammal, J. Phys. (Paris) 45, 191 (1984), and by M. Arai, T. Tokihiro, T. Fujiwara, and M. Kohmoto, Phys. Rev. B 38, 1621 (1988).

${ }^{20}$ For a review, see M. F. Barnsley, Fractals Everywhere (Academic, San Diego, 1988).

${ }^{21}$ That approximative Green's-function schemes catch little of the intricacy of the model's physical properties has been shown recently by M. P. Barbosa, R. F. S. Andrade, and H. J. Schellnhuber, in Proceedings of the 4th Brazilian School on Semiconductors, edited by A. Chaves (World Scientific, Singapore, 1990).

${ }^{22}$ Many authors have demonstrated the usefulness of RG techniques for evaluating the critical properties of self-similar structures. See, e.g., Y. Gefen, B. B. Mandelbrot, and A. Aharony, Phys. Rev. Lett. 45, 855 (1980). 